\title{
Application of the acousto-optic effect to pressure measurements in ultrasound fields in water using a laser vibrometer
}

\author{
J. M. Buick ${ }^{\text {a) }}$ \\ Physics and Electronics, School of Biological, Biomedical and Molecular Sciences, \\ University of New England, NSW 2351, Australia \\ J. A. Cosgrove, P.-A. Douissard, and C. A. Greated \\ School of Physics, The University of Edinburgh, EH9 3JZ, Scotland, United Kingdom \\ B. Gilabert \\ Départment de Génie Mécanique, ENS de Cachan, 94235 Cachan Cedex, France
}

(Received 8 January 2004; accepted 2 July 2004; published 20 September 2004)

\begin{abstract}
A non-intrusive measuring technique, applied to sensing and measuring acoustic waves at ultrasonic frequencies is considered. The method is optically based and so does not interfere with the ultrasound field. The measurement procedure relies on the acousto-optic effect, that is the change in refractive index which occurs with changing pressure in the ultrasound field. This change in refractive index is detected through the change in the path length of a laser beam propagating through the region of interest. Typically these changes are small corresponding to a physical change of the order of $10^{-6} \mathrm{~m}$. Fourier analysis is used to separate the component of the signal corresponding to the pressure variation from background noise and vibrations which can be dominant. Application of the technique is illustrated for an underwater ultrasound transducer. Measurements are made using the optical technique and compared to measurements taken with a hydrophone. The effectiveness of the optical measuring technique is discussed. It is shown that the laser vibrometer produces a good estimation of the mean beam pressure provided an estimation of the beam width is available, a restriction which is often satisfied; and the acoustic field can be assumed to be approximately constant across the beam. () 2004 American Institute of Physics. [DOI: 10.1063/1.1790556]
\end{abstract}

\section{INTRODUCTION}

Sound waves occur in a large variety of natural and technological areas and have relevance in, for example, musical acoustics, speech and hearing research, diagnostic imaging, physiotherapy applications, sonochemistry and sonar. A sound wave is generated when the fluid close to a vibrating surface is compressed and expanded. Conventionally a microphone or hydrophone is used to measure the pressure in a sound field. Alternatively the laser Doppler anemometry $(\mathrm{LDA})^{1,2}$ technique can be applied. This measures the acoustic particle velocity directly and in principle does not require calibration. It has the further advantage over a microphone that it is an optical method and so is nonintrusive. It is well know that the refractive index of a fluid is dependent on the fluid density. ${ }^{3,4}$ This means that when an acoustic wave propagates through a fluid the refractive index of the fluid changes as a function of both time and space, altering the optical properties of the fluid. This acousto-optic effect has been observed in laser measurement techniques such as $\mathrm{LDA}^{3,5,6}$ when applied to measuring fluid velocities in an acoustic field. In such cases the acousto-optic effect is undesirable since it introduces an error into the fluid measurement. In this article we examine a measurement technique which utilizes the acousto-optic effect and an application of

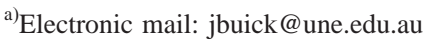

the method to ultrasound waves in water is examined. An alternative, nonintrusive measurement technique which also utilizes the acousto-optic technique has recently been proposed by Harland and co-workers. ${ }^{7,8}$ The two methods are compared in Sec. IV.

\section{ACOUSTO-OPTIC THEORY}

We first wish to investigate the theoretical aspects involved with aligning the laser. Consider a traveling wave with angular frequency $\omega$ and wave vector $\mathbf{k}=k \mathbf{e}_{y}$, where $\mathbf{e}_{y}$ is a unit vector in the $y$ direction, see Fig. 1. The refractive index of the fluid is given by ${ }^{3,9}$

$$
n(\mathbf{x}, t)=n_{0}-\delta n(\mathbf{x}, t),
$$

where

$$
\delta n=\Delta n \sin (\mathbf{k} \cdot \mathbf{x}+\omega t)=\Delta n \sin (k y+\omega t),
$$

$n_{0}$ is the refractive index of the fluid when there is no acoustic wave present and

$$
\Delta n=\frac{a k \sqrt{M \rho c^{3}}}{2} \text {. }
$$

Here $M$ is the material parameter, $\rho$ is the fluid density, $a$ is the amplitude of the acoustic wave, and $c$ is the speed of sound in the fluid. The pressure and the refractive index are related by, see e.g., Refs. 3 and 4, 


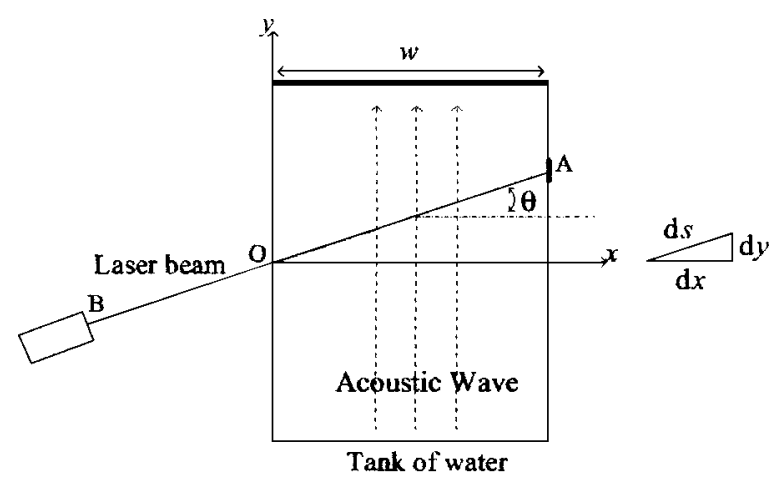

FIG. 1. Schematic diagram of the wave tank and laser beam.

$$
\Delta p(x)=2 \sqrt{\frac{\rho c}{M}} \Delta n(x),
$$

where the first-order approximation that the fluid pressure and density are linearly related has been assumed. Consider a laser beam entering a tank of water of width $w$ at an angle $\theta$ to the $x$ axis as shown in Fig. 1 and assume that a plane traveling wave is propagating in the tank. When a laser beam encounters changes in the refractive medium it will generally travel in a curved path. If we assume that the beam travels in a straight line $\gamma$ given by $y=\tan (\theta) x$ (true for small $\theta$ since the refractive index varies only as a function of $y$ for the traveling wave shown in Fig. 1), then the change in the optical path length of the laser traveling from $O$ to $A$ is

$$
\delta=-\Delta n \int_{\gamma} \sin (k y+\omega t) \mathrm{d} s \equiv-\Delta n I,
$$

where $d s=d y / \sin (\theta)$. Integrating gives

$$
\begin{aligned}
I & =\frac{2}{k \sin (\theta)} \sin \left(\frac{k w \tan (\theta)}{2}\right) \sin \left(\frac{k w \tan (\theta)}{2}+\omega t\right) \\
& \equiv g(\theta ; k, w) \sin \left(\frac{k w \tan (\theta)}{2}+\omega t\right) .
\end{aligned}
$$

That is, for a fixed frequency and tank width, $\delta$ varies sinusoidally at the frequency of the acoustic wave with an amplitude which depends on $\theta$ through $g(\theta)$. The relative phase of $\delta$ and the acoustic wave also depends on $\theta$ through the term $k w \tan (\theta) / 2$. Figure 2 shows the variation of $g(\theta)$ for acoustic waves with frequencies $f=10,22$ and $40 \mathrm{kHz}$ and $w=0.45 \mathrm{~m}$. This shows the measurement of the optical path length is sensitive to the angle $\theta$. The sensitivity is seen to depend on the frequency of the acoustic waves for a fixed tank width. In the remainder of this article a frequency of $22 \mathrm{kHz}$ and tank width $w=0.45 \mathrm{~m}$ will be used and only $\theta$ $=0$ will be considered. In this case an error of $1^{\circ}$ in $\theta$ will only give an error of $2 \%$ in $\delta$, however a variation of $5^{\circ}$ will give an error of over $40 \%$ in $\delta$. Therefore it is necessary to ensure that the error in aligning the laser vibrometer at right angles to the direction of propagation of the ultrasound field is small to ensure the accuracy of the measurement of the optical path length. This becomes increasingly important as the frequency of the ultrasound is increased. The reduction in the value of $g$ for $\theta \neq 0$ is due to the laser beam propagating through regions of both high and low refractive index which

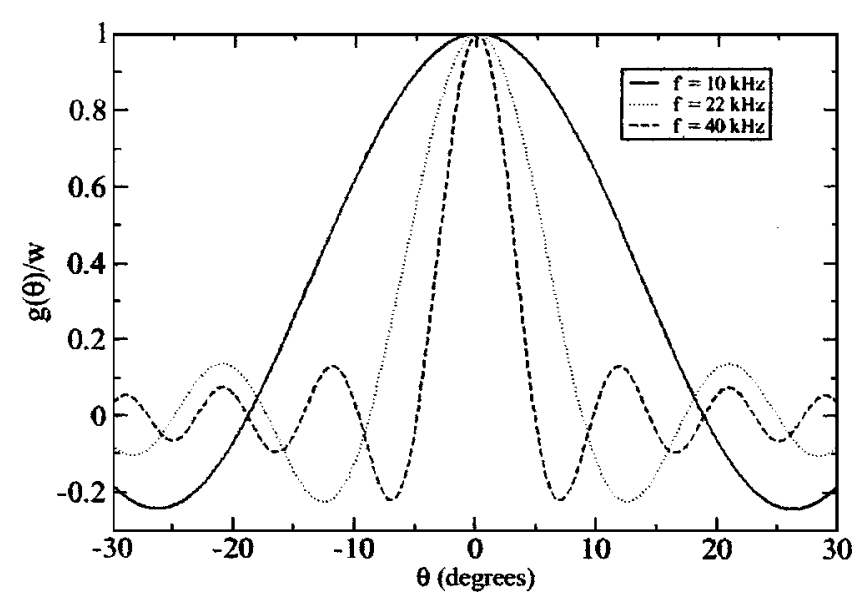

FIG. 2. The variation of $g(\theta)$ with $\theta$ plotted frequencies of 10,22 , and $40 \mathrm{kHz}$, for $w=0.45 \mathrm{~m}$.

act to cancel each other out in terms of a measured change in the overall path length. This can be visualized in Fig. 3 which shows the refractive index field for a $22 \mathrm{kHz}$ acoustic wave in waver. Also shown are the laser beam paths for $\theta$ $=1^{\circ}, 5^{\circ}$, and $10^{\circ}$. For $\theta=1^{\circ}$ (dashed line) the refractive index is similar along the whole of the laser beam giving $g(\theta)$ $\simeq 1$. When the angle of the laser beam is increased to $5^{\circ}$ there is a significant change in the refractive index over the length of the beam accounting for the $40 \%$ error in $g(\theta)$ indicated in Fig. 2. The value of the refractive index over the first half of the $\theta=10^{\circ}$ line is approximately canceled by the value along the second half; thus the value of $g(\theta)$ in Fig. 2 is approximately zero. It is evident from Fig. 3 that the error will increase with increasing $w$. It is clear from Eq. (6) that the error will increase linearly with $w$ in the low $\theta$ limit.

\section{EXPERIMENTAL SYSTEM}

The experimental setup is shown in Fig. 4. A signal generator and amplifier were used to drive the ultrasound device which was fixed at one end of a glass tank filled with water

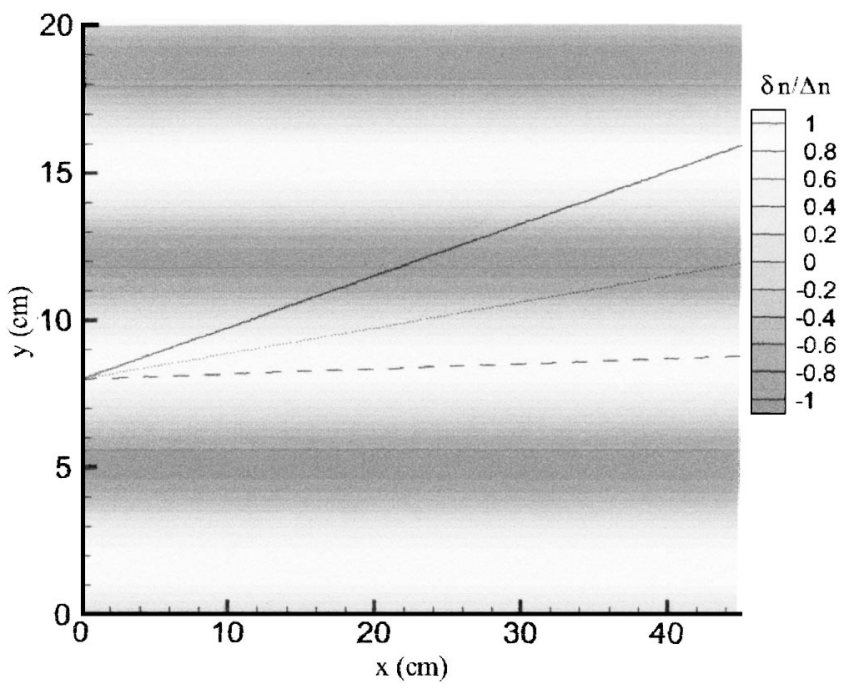

FIG. 3. The path of the laser beam through the refractive index change field for a $22 \mathrm{kHz}$ ultrasound wave in water with $\theta=1^{\circ}$ (dashed), $\theta=5^{\circ}$ (dotted), and $\theta=10^{\circ}$ (dotted). 


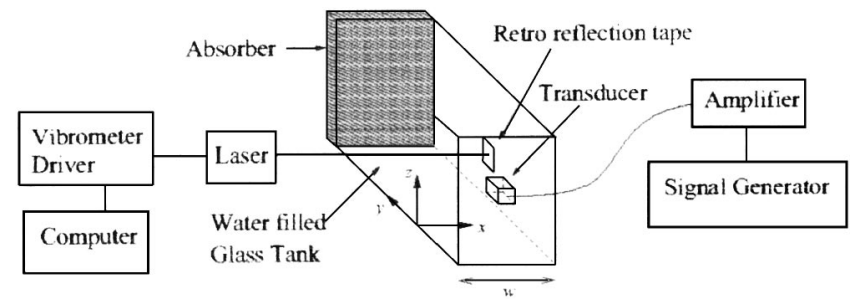

FIG. 4. The experimental setup. The coordinate system was selected such that $y-0$ corresponds to the front of the transducer.

to a height of $40 \mathrm{~cm}$. The transducer was placed at one end of the tank at mid-water depth with the transducer head parallel to the wall, as shown in Fig. 4. The tank was $1.2 \mathrm{~m}$ long, $0.45 \mathrm{~m}$ wide, and $0.65 \mathrm{~m}$ high. Absorbing polyurethane rubber ${ }^{10}$ was placed at the other end of the tank. This had the effect of reducing, but not totally eliminating, reflections at the wall. The acoustic field produced depends on the geometry of the tank as well as the properties of the acoustic source. Partial reflections from the opposite end of the tank as well as reflections from the side walls and the free surface result in a complex wave pattern which is considerably different from the free-field acoustic field. Here we are concerned with the measurement technique rather than the details of the acoustic field. The laser beam from a Polytec laser vibrometer OFV-3001-23/OFV-303 system was directed through the tank onto a piece of retro-reflective tape which was stuck to the outside of the far wall. Alternatively any fixed reflective surface could be used without altering the measured optical path length. The surface of the retroreflective tape consists of small hemispheres which reflect the laser light over a large solid angle approaching $2 \pi$ sr. As well as this broad reflection there is a distinct reflected beam with an angle of reflection relative to the surface normal which is equal to the angle of incidence. By ensuring that the reflected beam is consistent with the incident beam the laser can be aligned normal to the tank wall. The head of the ultrasound transducer was then adjusted by rotating it in the $x-y$ plane to achieve $\theta=0$. This was taken to be the angle at which the measured change in the optical path length was maximum. In practical situations where the transducer is fixed it is possible to alter the angle of the laser beam. Since the retro-reflective tape reflects over a large solid angle the incident beam does not need to be normal to the surface of the retro-reflective tape.

The laser vibrometer is designed to make independent measurements of the velocity and displacement of a vibrating surface. The former is achieved by analyzing the Doppler shift, however it is the displacement measurement which is of interest here. This is obtained in the vibrometer control box by measuring the change in the optical path length of the beam. The beam is circularly polarized and it is the change in the phase of polarization of the returning beam which is measured and converted by the control box to give the optical path length. This calculation is performed internally by the system and cannot be influenced by the operator. A discussion of measurement techniques, including the approach utilized in the Polytec laser vibrometer, is given by Egan et $a l .{ }^{11}$ In its normal application the path length changes due to

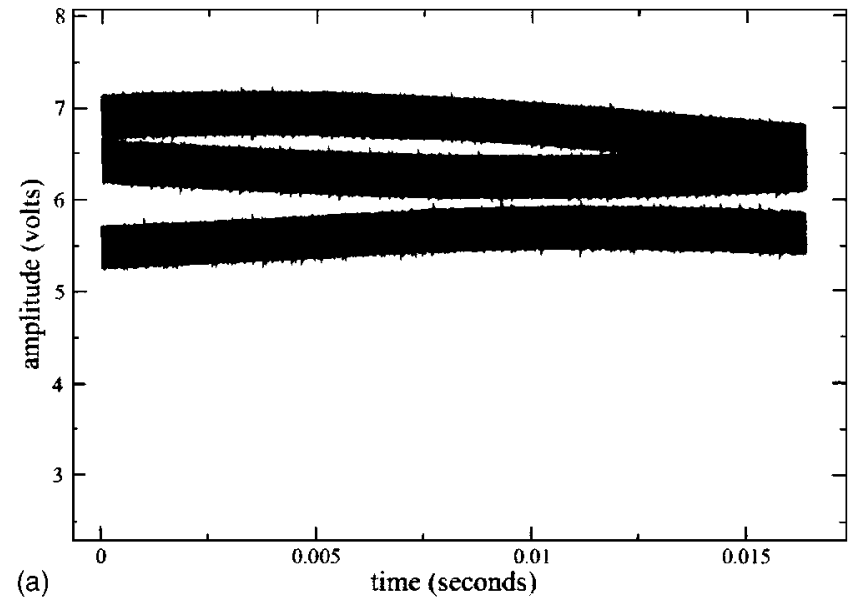

(a)

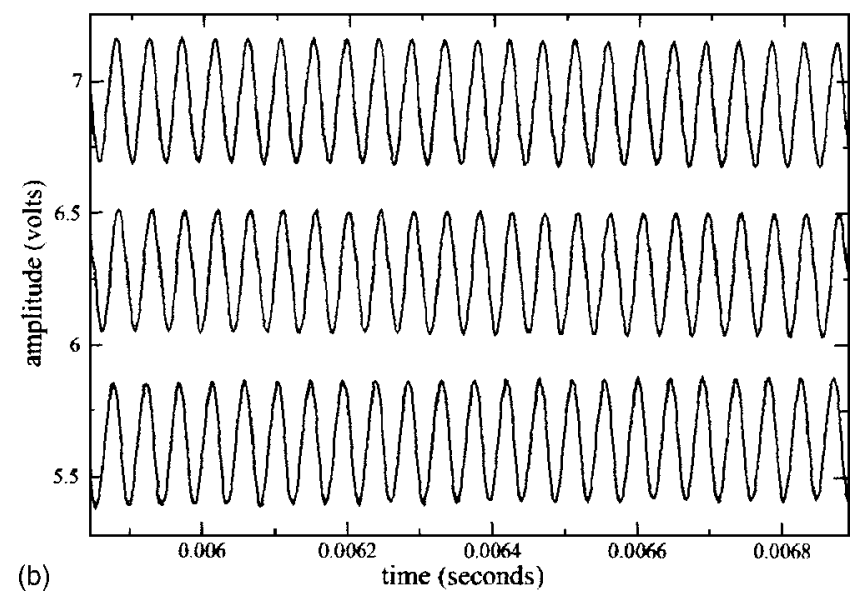

FIG. 5. Output signal from the laser vibrometer, magnified in (b).

the motion of the reflected surface, however here the retroreflective tape on the outside wall of the tank is assumed to be stationary; the change in the optical path length which is measured by the laser vibrometer is due to a change in the refractive index inside the fluid. Figures 5(a) and 5(b) show a typical output from the laser vibrometer with a sensitivity of $0.5 \mu \mathrm{m} \mathrm{V}^{-1}$. The three lines correspond to samples of the signal taken $0.1 \mathrm{~s}$ apart. Two features of the signal are evident: Fig. 5(b) indicates that on a relatively short time-scale of order tens of periods the path length is changing in a sinusoidal manner while Fig. 5(a) shows the envelope of these oscillations varying on a longer time scale greater than $0.01 \mathrm{~s}$. Evidently the laser vibrometer is detecting both the variations in the path length due to the acousto-optic effect with a frequency of $22 \mathrm{kHz}$ and also variations in the position of the tank wall relative to the laser head due to, for example, floor vibrations, with a frequency of the order of tens of hertz. It should be noted that the displacement measurements from the laser vibrometer are relative: vibration of the object or the laser head will give the same reading. It is also important to note that the output corresponds to half of the total change in the optical path length of the laser beam as it travels from the laser head to the reflection point and back to the laser head, as would be required in a displacement measurement. The speed of light is significantly larger than the speed of sound such that the acoustic field will have changed negligibly in the time between the outgoing and reflected laser beam. 

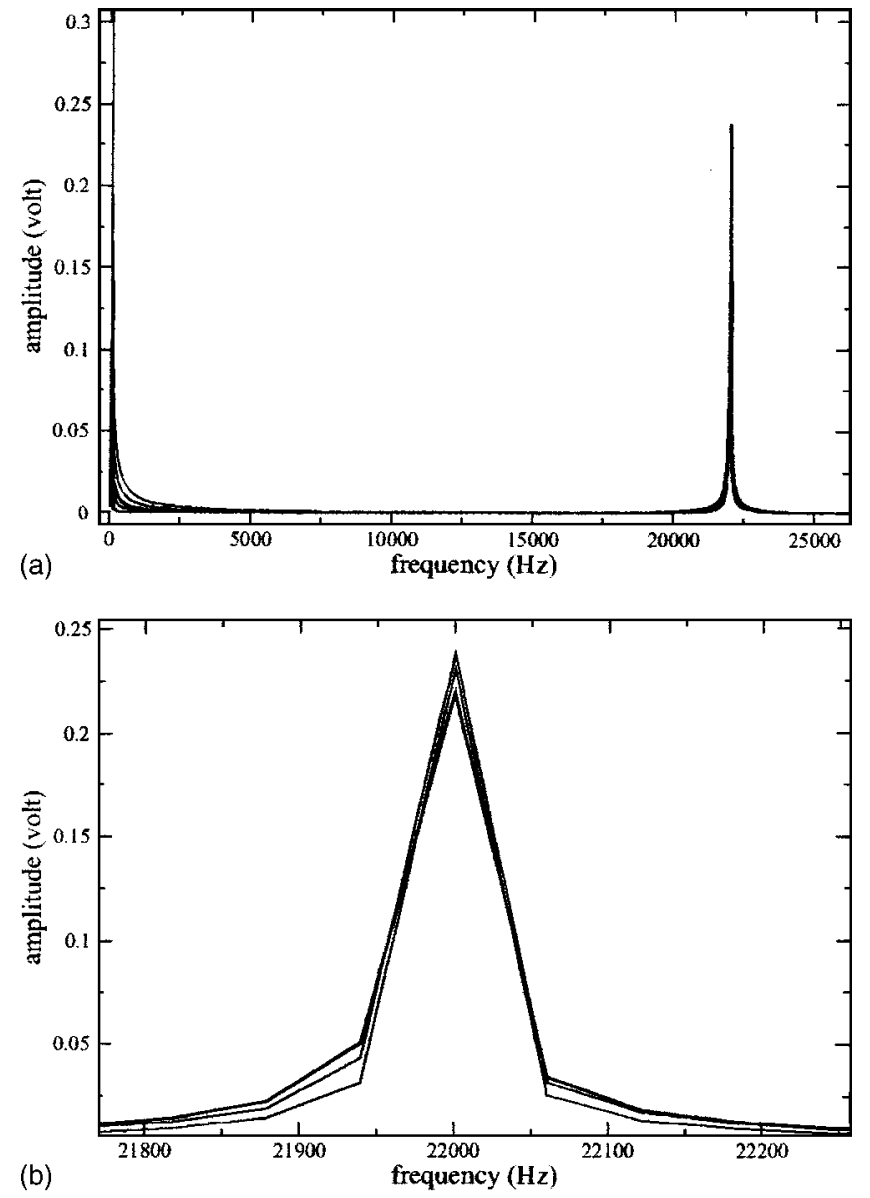

FIG. 6. Frequency components of the output signal from the laser vibrometer, magnified in (b).

The variation between the curves in Fig. 5(a) corresponds to a displacement larger than the amplitude of the variations due to the acousto-optic phenomenon. In the measurements presented here external vibrations were minimized by setting the apparatus on a heavy optical table. If the technique were applied in an industrial setting this might not be possible and the low frequency vibration might be larger. However, since the vibrations are at a much lower frequency than the ultrasound, the two signals can be effectively separated using Fourier analysis. This is shown in Figs. 6(a) and 6(b) which indicate the frequency components of repeated measurements. In Fig. 6(a) the low frequency vibrations and the ultrasound frequencies are seen to be clearly separated. Figure 6(b) shows five repeated measurements each showing a peak at $22 \mathrm{kHz}$. Three of the results (corresponding to the smallest peak) are virtually identical, however the other peak values are slightly different giving a variation of approximately $10 \%$ in the voltage reading and hence in the change in optical path length. Variations were observed when a short time series was taken. These are due to problems such as signal drop-out which can occur, for example, due to speckle noise. A consistent value can be obtained by taking the average over a number of short measurements and this approach was taken for the measurements presented in the remainder of this article.

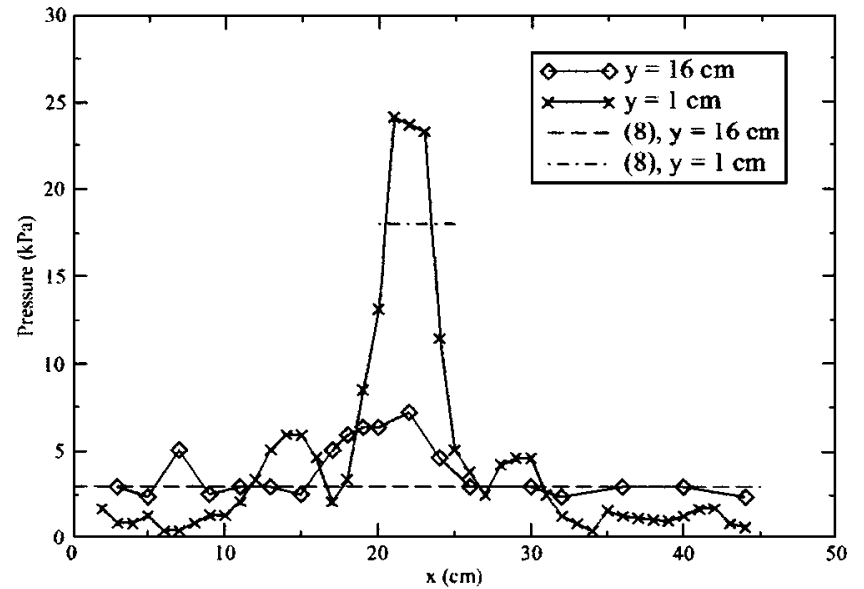

FIG. 7. Hydrophone pressure measurements and the pressure calculated from Eq. (8).

\section{RESULTS AND DISCUSSION}

A Brüel \& Kjær 8103 hydrophone with a frequency range of $0.1 \mathrm{~Hz}-180 \mathrm{kHz}$ was used to measure the pressure at points within the tank to enable a comparison to be made with the laser vibrometer measurements. Figure 7 shows the hydrophone measurements just in front of the transducer ( $y$ $=1 \mathrm{~cm})$ and also at $y=16 \mathrm{~cm}$, which corresponds to the position where the ultrasound beam reaches the edges of the tank (from the manufacturers data sheet). Applying the laser vibrometer beam along these lines $(y=1 \mathrm{~cm}$ and $y=16)$, the change in the optical path length due to the acoustic field was measured as $\delta=0.147$ and $0.124 \mu \mathrm{m}$, respectively. Now the pressure and the refractive index along the path of the laser vibrometer are related by Eq. (4), see, e.g., Refs. 3 and 4, where $M=160 \times 10^{-15} \mathrm{~s}^{3} \mathrm{~kg}^{-3},{ }^{12,13} \rho=1000 \mathrm{~kg} \mathrm{~m}^{-3}$ and $\mathrm{c}$ $=1500 \mathrm{~m} \mathrm{~s}^{-1}$. Now the change in the optical path length measured by the laser vibrometer is given by

$$
\delta=\int_{x=x_{0}}^{x=x_{0}+l} \Delta n d x,
$$

where $x=x_{0}$ and $x=x_{0}+l$ correspond to the limits of the acoustic field. If there is some information about the width of the acoustic beam, then it is possible to make an estimate of the pressure based on the assumption that it is approximately constant. The validity of such an assumption will vary depending on the details of the acoustic source and the system geometry; in this case the validity can be assessed by considering Fig. 7. In this case

$$
\Delta p=2 \sqrt{\frac{\rho c}{M}} \frac{\delta}{l} .
$$

For example at $y=1 \mathrm{~cm}$ we expect $l$ to be approximately the diameter of the source, $l \simeq 0.05 \mathrm{~m}$ in which case we get $\Delta p$ $\simeq 18 \mathrm{kPa}$ in the central region. Similarly at $y=16 \mathrm{~cm}$ we have $l \simeq w$, the tank width, which gives $\Delta p \simeq 3 \mathrm{kPa}$, which is similar to the mean value in Fig. 7. The calculated values are plotted in Fig. 7 indicating the level of agreement.

It has been shown that, provided there is an estimate of the width of the beam and the signal can be assumed to be 
approximately constant over its width, it is possible to obtain a reasonable approximation for the mean acoustic pressure within the beam. It is, however, often desirable to obtain a pressure reading at some position in the fluid. For such measurements the application of the laser vibrometer is limited since it acts to integrate the pressure over the entire laser path. One application could be to measure the acoustic pressure at a single critical point within a closed system where no pressure measuring device can be inserted. In this case the laser vibrometer could be applied provided a prior calibration of the instrument was performed. Such a calibration could be performed by fixing the laser path and measuring the change in optical path length as a function of pressure over the operating range. The pressure measurements being obtained, for example, by a pressure transducer prior to the system being sealed, possibly during manufacture.

An alternative measurement technique, laser Doppler velocimetry (LDV), which also detects the acoustic pressure through the change in refractive index has recently been proposed by Harland and co-workers. ${ }^{7,8}$ The LDV technique detects the rate of change of the total optical path length, $L$, with respect to time. This can be expressed, in the notation of this article, as ${ }^{8}$

$$
\frac{d L}{d t}=2 \frac{d\left(\int_{0}^{x} n(t) d x\right)}{d t} .
$$

Noting that $d L / d t \equiv d \delta / d t$ and that the integral is only timedependent between $x=x_{0}$ and $x=x_{0}+l$ this can be rewritten as

$$
\frac{d \delta}{d t}=2 \frac{d\left(\int_{x_{0}}^{x_{0}+l} n(t) d x\right)}{d t},
$$

which can be compared with Eq. (7). We note that the factor "2" in Eq. (10) is not required in Eq. (7) since it is accounted for in the laser vibrometer output (the distance a vibrating object has moved is twice the change in the optical path length). LDV determines the value of $d L / d t$ by detecting the Doppler shift in the reflected laser beam. This must then be integrated with respect to time to obtain the optical path length. The laser vibrometer employed in the present study determines the optical path length independently of the Doppler shift and thus eliminates the need to perform a numeri- cal time integration of the acquired data and the associated errors. The value of $\delta$ is determined by the polarity phase of the reflected beam which is circularly polarized. We note that in Ref. 8 the LDV results are presented in terms of $d L / d t$ and no attempt is made to recover $L$ or $\delta$ or the acoustic pressure. In the measurements presented here $\delta$ is measured directly.

Both LDV and the technique described here have been applied to ultrasound waves in water with a frequency $O\left(10^{4}\right) \mathrm{Hz}$. Now $O(d L / d t)=\omega O(\delta)$ as can be seen when comparing the results presented here and those in Ref. 8. Provided the laser vibrometer has sufficient resolution to measure the change in optical path length, $\delta$ the magnitude is not important. External vibrations in the system which introduce errors into the measurements are typically of the order of tens of hertz. This means that the relative magnitude of the external vibrations to the desired signal is greater by a factor $O\left(10^{3}\right)$ when $\delta$ is measured directly compared to a measurement of $d L / d t$. The significant magnitude of the external vibrations can be seen in Figs. 5 and 6, however by applying Fourier analysis it is possible to obtain the amplitude of the variation with the desired frequency and hence a pressure measurement, as demonstrated here.

${ }^{1}$ K. J. Taylor, J. Acoust. Soc. Am. 59, 691 (1976).

${ }^{2}$ M. Campbell, J. A. Cosgrove, C. A. Greated, S. Jack, and D. Rockliff, Opt. Laser Technol. 32, 629 (2000).

${ }^{3}$ S. H. Jack, D. B. Hann, and C. A. Greated, Rev. Sci. Instrum. 69, 4074 (1998).

${ }^{4}$ L. E. Kinsler, A. R. Frey, A. B. Coppens, and J. V. Sanders, Fundamentals of Acoustics, 3rd ed. (Wiley, New York, 1982).

${ }^{5}$ S. H. Jack, D. B. Hann, and C. A. Greated, Meas. Sci. Technol. 10, 1279 (1999).

${ }^{6}$ R. I. Crickmore, S. H. Jack, D. B. Hann, and C. A. Greated, Opt. Laser Technol. 31, 85 (1999).

${ }^{7}$ A. R. Harland, PhD thesis, University of Loughborough, 2002.

${ }^{8}$ A. R. Harland, J. N. Petzing, and J. R. Tyrer, J. Sound Vib. 252, 169 (2002).

${ }^{9}$ W. B. Leigh, Devices for Optoelectronics (Marcel Dekker, New York, 1996).

${ }^{10}$ Acoustic absorber type F28: National Physical Laboratory, Teddington, UK.

${ }^{11}$ D. A. Egan, S. W. James, and R. P. Tatam, Meas. Sci. Technol. 8, 343 (1997).

${ }^{12}$ R. W. Dixon, J. Appl. Phys. 38, 5149 (1967).

${ }^{13}$ D. A. Minnow, IEEE J. Quantum Electron. 6, 223 (1970). 